\title{
Minimal surgery achieved good visual acuity in selected patients with magnetic intravitreal foreign body and traumatic cataract
}

\author{
Zhitao Su, Panpan Ye, Jijian Lin, Li Zhang and Xiaodan Huang*
}

\begin{abstract}
Background: To explore minimal surgery in selected patients with intravitreal foreign body (IVFD) and traumatic cataract. Methods: Twelve eyes of 12 patients with small ferrous IVFD and traumatic cataract without endophthalmitis, retinal injury and secondary glaucoma, between September 2015 and March 2017 were retrospectively analyzed. Primary removal of IVFD was performed by external magnetic extraction through the pars plana incision. Secondary removal of traumatic cataract by phacoemulsification and intraocular lens (IOL) implantation with or without anterior vitrectomy were performed. Patients were followed up at 1 day, 1 week, 1 month, 3 months, 6 months and 12 months after surgery.

Results: All patients were male with a mean age of 32 years old. All IVFDs were successfully removed without retinal injury. Two to 6 months later, the traumatic cataract was successfully removed by phacoemulsification combined with IOL implantation in the capsule bag in 10 patients. Anterior vitrectomy was implied in 2 patients with large posterior capsule rupture, and the IOLs were placed in the ciliary sulcus. Best-corrected visual acuity ranged from hand movement to 20/100 before surgery and improved ranging from 20/32 to 20/20 at the final follow-up. The IOLs were well centered. Complications such as secondary glaucoma, endophthalmitis and retinal detachment were not found.
\end{abstract}

Conclusions: Primary removal of small ferrous IVFD by external magnetic extraction followed by secondary cataract removal and IOL implantation is an appropriate choice. Minimal surgery may obtain good visual outcome without complications in selected patients.

Keywords: Intravitreal foreign body, Traumatic cataract surgery, IOL implantation, Minimal surgery

\section{Background}

Among patients with posterior segment intraocular foreign body (IOFB) and traumatic cataract, visual rehabilitation poses a unique challenge to ophthalmic surgeons. With the development of surgical techniques and instruments, there is an increasing trend toward performing pars plana vitrectomy (PPV) and simultaneous cataract extraction in the management of these patients [1-3]. However, various potential complications of PPV have been reported [4-6]. Ferrous intravitreal foreign body

\footnotetext{
* Correspondence: huangxiaodan@zju.edu.cn

Eye Center, Second Affiliated Hospital, School of Medicine, Zhejiang University, No. 88 Jiefang Rd, Hangzhou 310009, China
}

(IVFB), which did not damage the retina can be successfully removed by external magnetic extraction without PPV [7]. In the absence of increased intraocular pressure (IOP) or severe inflammatory reaction in traumatic cataract with posterior capsule rupture, delaying surgery would have allowed a more favorable intraocular lens (IOL) implantation in capsular bag with a better visual prognosis and lesser complications after control of inflammation and fibrosis of the capsule rupture [8].

To explore the possibility of minimal surgery in selected patients with small ferrous IVFD and traumatic cataract, here we report a series of cases with primary removal of IVFD by external magnetic extraction and

(c) The Author(s). 2019 Open Access This article is distributed under the terms of the Creative Commons Attribution 4.0 International License (http://creativecommons.org/licenses/by/4.0/), which permits unrestricted use, distribution, and reproduction in any medium, provided you give appropriate credit to the original author(s) and the source, provide a link to the Creative Commons license, and indicate if changes were made. The Creative Commons Public Domain Dedication waiver (http://creativecommons.org/publicdomain/zero/1.0/) applies to the data made available in this article, unless otherwise stated. 
secondary cataract removal combined with IOL implantation without PPV, which obtained good visual outcome without postoperative complications.

\section{Methods}

The study comprised penetrating eyes with paracentral or peripheral self-sealing corneal penetrating wound, traumatic cataract and ferrous IVFD from September 2015 to March 2017. Eyes with endophthalmitis, retinal injury, vitreous hemorrhage, lens materials into the anterior chamber or vitreous cavity, active inflammation, or associated glaucoma were excluded from the study. The study was performed in accordance with the ethical standards stated in the Declaration of Helsinki.

A thorough history was collected from the patients. Best-corrected visual acuity (BCVA), slit-lamp examination, IOP, binocular indirect ophthalmoscopy wherever possible, B-scan ultrasonography, and orbital computed tomography were performed to evaluate the eye injuries.

After confirming that there was no secondary glaucoma, endophthalmitis, or retinal injury and identification of the metallic-like foreign body suspended in the vitreous cavity, primary removal of IVFD was performed by direct external magnetic extraction. The pars plana, adjacent to the foreign body, was exposed by opening the bulbar conjunctiva. Preplacing sclerotomy suture was performed to allow quick closure once the foreign body was removed. A sclerotomy, $4.0 \mathrm{~mm}$ from the corneal limbus, was made. The choroid was diathermized and incised. The rare earth magnet was placed at the sclerotomy site and the foreign body was removed. The vitreous, if attached to the foreign body, was cut before the foreign body left the sclera. The sclerotomy and the open conjunctiva were closed by $8-0$ absorbable polypropylene suture (W9560, Johnson \& Johnson). After removal of the IVFD, patient received $0.5 \%$ levofloxacin eye drops, $1 \%$ prednisolone acetate eye drops and $1 \%$ pranoprofen eye drops 2-8 times a day for 4 weeks, and $0.5 \mathrm{~g}$ levofloxacin tablet a day for 4 days. Patients were followed up at 1 day, 3 days, 1 week, 1 month after surgery. Slit-lamp examination, IOP, binocular indirect ophthalmoscopy wherever possible, and B-scan ultrasonography were performed to exclude secondary glaucoma, endophthalmitis or retinal detachment.

Two to 5 months after primary removal of the IVFD, the traumatic cataract was removed by phacoemulsification and/or aspiration (Bausch \& Lomb, Stellaris). In white cataract, for a better view of the anterior capsule, $0.5 \%$ indocyanine green was applied for the staining of the anterior capsule. To prevent an enlargement of the posterior capsule rupture and vitreous prolapse, bottle height was set at $80 \mathrm{~cm}$ and maximal vacuum was set at $250 \mathrm{mmHg}$, viscoelastic (Healon, Johnson \& Johnson Vision) was injected into the anterior chamber before withdrawal of the ultrasonic or irrigation/aspiration handle. If necessary, anterior vitreous vitrectomy was applied. IOLs (8 from AMO, AR40e and 4 from AMO, ZCB00) were placed in the capsule bag or in the ciliary sulcus. Postoperatively, patient received $0.5 \%$ levofloxacin eye drops 4 times a day for 2 weeks, $1 \%$ prednisolone acetate eye drops and 1\% pranoprofen eye drops 2-4 times a day for 8 weeks. Patients were followed up at 1 day, 1 week, 1 month, 3 months, 6 months and 12 months after surgery.

\section{Results}

Twelve patients (all men) were included in the study. The means of injury was hammering metal during occupational activities. The characteristics and outcomes are shown in Table 1 . The average age was 32 years (range 19-46 years). The foreign bodies passed through cornea, iris, lens, and finally localized in the vitreous cavity. Four eyes developed localized cataract (Patient 6 with localized cataract was shown in Fig. 1), which interfered with visual axis, and 8 eyes developed total cataract (Patient 9 with total cataract was shown in Fig. 2).

All cases presented self-sealing corneal penetrating wound, mild anterior chamber reaction, small iris defect and/or posterior synechia, and traumatic cataract without lens material into the anterior chamber or vitreous cavity. No secondary glaucoma was found. Metallic-like foreign bodies suspended in the vitreous cavity were identified by B-scan ultrasonography or orbital computed tomography. Small metallic-like IVFD was observed by indirect ophthalmoscopy in 2 patients with localized cataract. No retinal injury was found by B-scan ultrasonography.

BCVA ranged from hand movement to 20/100 before surgery. Time between injury and primary removal of foreign body was $47 \mathrm{~h}$ on average (range 20-92 h). All foreign bodies were successfully removed by external magnetic extraction through the pars plana incision. The mean size of the foreign bodies was $1.3 \mathrm{~mm}$ in width (range $1.0-1.5 \mathrm{~mm}$ ) and $2.0 \mathrm{~mm}$ in length (range 1.5$3.0 \mathrm{~mm}$ ). During follow-up, none of the secondary glaucoma, endophthalmitis or retinal detachment was found.

The average time between primary removal of foreign body and secondary traumatic cataract surgery was 107 days (range 79-162 days). For a better view of the anterior capsule, indocyanine green staining of the capsule was used in 8 patients with total cataract; anterior capsulorhexis was successfully performed in all cases. The cataract was removed by phacoemulsification and/or aspiration without significant enlargement of posterior capsule rupture in 10 patients, and the IOLs (6 from AMO, AR40e and 4 from AMO, ZCB00) were placed in the capsule bag. Enlargement of the posterior rupture was found in 2 patients (patient 3 and 10) with relatively large foreign bodies, anterior vitrectomy was performed 
Table 1 Demographics data and outcomes of all patients

\begin{tabular}{|c|c|c|c|c|c|c|c|c|c|c|}
\hline \multirow[t]{2}{*}{$\mathrm{NO}$} & \multirow[t]{2}{*}{ Age (ys) } & \multirow[t]{2}{*}{ Entry site } & \multirow[t]{2}{*}{ Cataract } & \multirow{2}{*}{$\begin{array}{l}\text { Size of } \\
\text { FD (mm) }\end{array}$} & \multirow{2}{*}{$\begin{array}{l}\text { Time to FD } \\
\text { removal }\end{array}$} & \multirow{2}{*}{$\begin{array}{l}\text { Time to } \\
\text { cataract } \\
\text { surgery }\end{array}$} & \multirow{2}{*}{$\begin{array}{l}\mathrm{IOL} \\
\text { position }\end{array}$} & \multirow[t]{2}{*}{ Follow up } & \multicolumn{2}{|l|}{ BCVA } \\
\hline & & & & & & & & & preo & final \\
\hline 1 & 34 & $\mathrm{C} / \mathrm{I} / \mathrm{L}$ & localized & $1.0 \times 1.5$ & 92 hs & $142 \mathrm{ds}$ & $C B$ & $15 \mathrm{~ms}$ & $20 / 200$ & $20 / 20$ \\
\hline 2 & 22 & $\mathrm{C} / \mathrm{I} / \mathrm{L}$ & localized & $1.0 \times 2.0$ & 68 hs & $130 \mathrm{ds}$ & $C B$ & $16 \mathrm{~ms}$ & $20 / 200$ & $20 / 25$ \\
\hline 3 & 30 & $\mathrm{C} / \mathrm{I} / \mathrm{L}$ & total & $1.5 \times 3.0$ & 38 hs & $94 \mathrm{ds}$ & CS & $18 \mathrm{~ms}$ & HM & $20 / 32$ \\
\hline 4 & 44 & $\mathrm{C} / \mathrm{I} / \mathrm{L}$ & total & $1.5 \times 2.0$ & $47 \mathrm{hs}$ & $98 \mathrm{ds}$ & $C B$ & $15 \mathrm{~ms}$ & FC & $20 / 25$ \\
\hline 5 & 46 & $\mathrm{C} / \mathrm{I} / \mathrm{L}$ & localized & $1.5 \times 1.5$ & 62 hs & $125 \mathrm{ds}$ & $C B$ & $18 \mathrm{~ms}$ & $20 / 125$ & $20 / 25$ \\
\hline 6 & 26 & $\mathrm{C} / \mathrm{I} / \mathrm{L}$ & localized & $1.0 \times 1.0$ & 70 hs & $162 \mathrm{ds}$ & $C B$ & $12 \mathrm{~ms}$ & $20 / 100$ & $20 / 20$ \\
\hline 7 & 24 & $\mathrm{C} / \mathrm{I} / \mathrm{L}$ & total & $1.5 \times 2.0$ & 20 hs & $79 \mathrm{ds}$ & CB & $15 \mathrm{~ms}$ & FC & $20 / 25$ \\
\hline 8 & 31 & $\mathrm{C} / \mathrm{I} / \mathrm{L}$ & total & $1.0 \times 2.5$ & 26 hs & $90 \mathrm{ds}$ & CB & $12 \mathrm{~ms}$ & $\mathrm{HM}$ & $20 / 32$ \\
\hline 9 & 19 & $\mathrm{C} / \mathrm{I} / \mathrm{L}$ & total & $1.5 \times 2.0$ & 40 hs & $88 \mathrm{ds}$ & CB & $12 \mathrm{~ms}$ & FC & $20 / 20$ \\
\hline 10 & 33 & $\mathrm{C} / \mathrm{I} / \mathrm{L}$ & total & $1.5 \times 2.5$ & 30 hs & 79 ds & CS & $12 \mathrm{~ms}$ & $\mathrm{HM}$ & $20 / 32$ \\
\hline 11 & 42 & $\mathrm{C} / \mathrm{I} / \mathrm{L}$ & total & $1.0 \times 2.0$ & $44 \mathrm{hs}$ & $68 \mathrm{ds}$ & CB & $20 \mathrm{~ms}$ & FC & $20 / 25$ \\
\hline 12 & 34 & $\mathrm{C} / \mathrm{I} / \mathrm{L}$ & total & $1.0 \times 1.5$ & 30 hs & $75 \mathrm{ds}$ & $C B$ & $10 \mathrm{~ms}$ & $20 / 200$ & $20 / 32$ \\
\hline
\end{tabular}

Abbreviations: ys years, $C$ cornea, $I$ iris, $L$ lens, $F D$ foreign body, hs hours, ds-days $I O L$ intraocular lens, $C B$ capsule bag, $C S$ ciliary sulcus, ms-months, $B C V A$ best corrected visual acuity, preo-preoperative, HM hand movement, FC finger counting

and the IOLs (AMO, AR40e) were placed in the ciliary sulcus.

The mean time of follow-up was 15 months (range 12-20 months). BCVA ranged from hand movement to $20 / 100$ before surgery and improved ranging from $20 / 32$ to $20 / 20$ at the final follow-up. The IOLs were well centered. Complications such as secondary glaucoma, endophthalmitis and retinal detachment were not found.

\section{Discussion}

The prognosis of traumatic eye injuries associated with IOFB and traumatic cataract varies greatly depending on
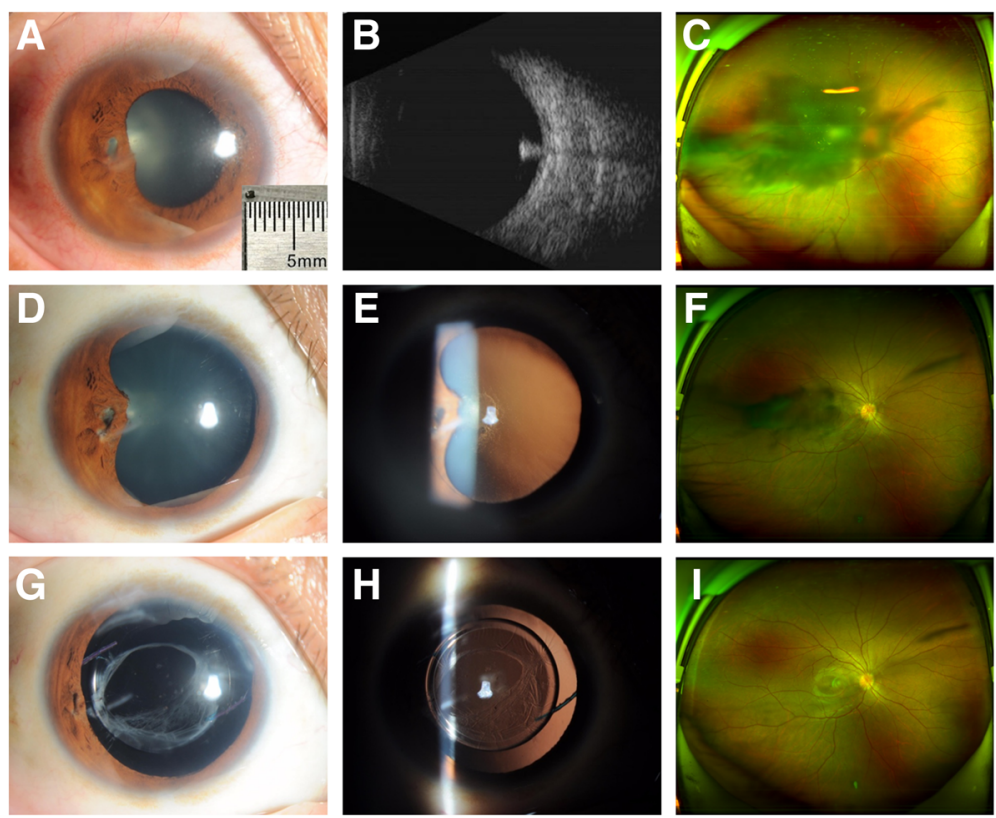

Fig. 1 Patient 6 with localized cataract. a: Anterior segment photograph revealed a paracentral self-sealing corneal penetrating wound at 8 o'clock position, iris defect and posterior synechia, and localized cataract involved the visual axis. The size of foreign body was $1.0 \mathrm{~mm}$ in width and $1.0 \mathrm{~mm}$ in length. $\mathbf{b}$ : A small metallic-like foreign body was identified by B-scan ultrasonography. $\mathbf{c}$ : A dense shadow appeared in the middle of visual field by scanning laser ophthalmoscopic image. $\mathbf{d}$, e: The traumatic cataract partially resolved 5 months after removal of the foreign body. f: A slight shadow appeared in the middle of visual field. $\mathbf{g}, \mathbf{h}$ : The IOL was well centered 12 months after secondary removal of traumatic cataract and implantation of IOL. i: A linear shadow caused by the corneal scar appeared in peripheral visual field 


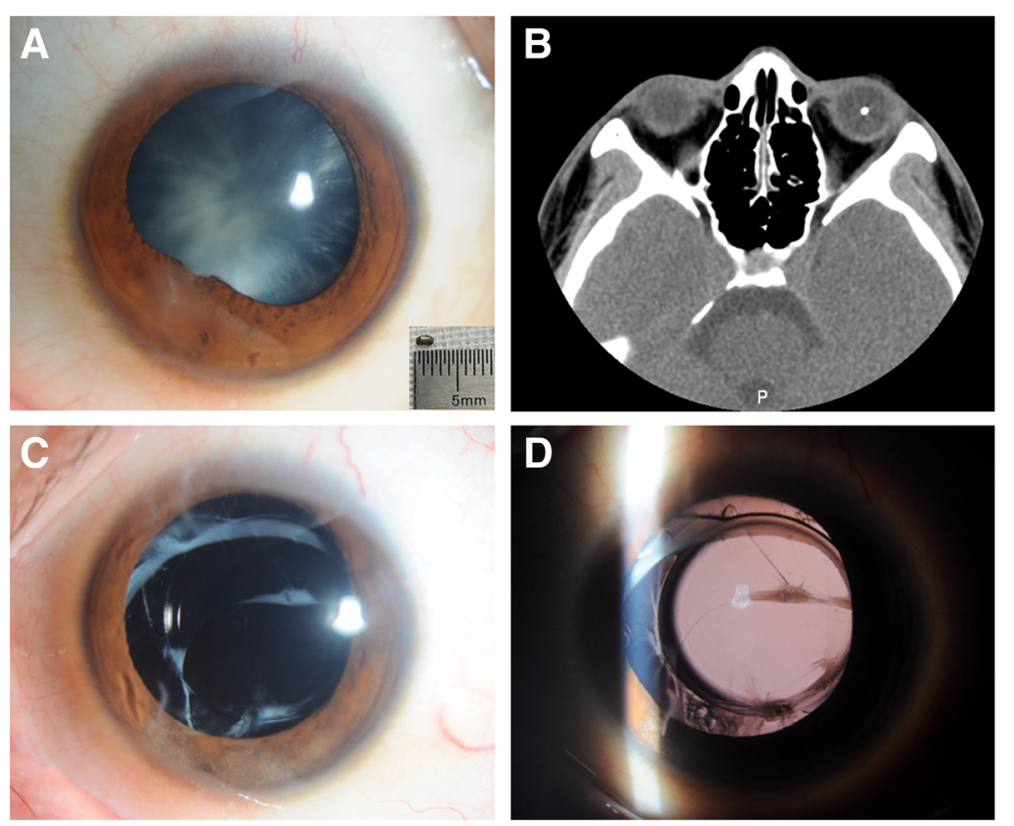

Fig. 2 Patient 9 with total cataract. a: Anterior segment photograph revealed a peripheral self-sealing corneal penetrating wound at 7 o'clock position, iris posterior synechia, and total cataract. The size of foreign body was $1.5 \mathrm{~mm}$ in width and $2.0 \mathrm{~mm}$ in length. b: A small metallic-like foreign body, suspended in the vitreous cavity, was identified by orbital computed tomography. c, d: The IOL was well centered 12 months after secondary removal of traumatic cataract and implantation of IOL

a number of factors, which include the time between trauma and IOFB extraction, initial visual acuity, entrance wound location, nature of IOFB, location of IOFB, preoperative retinal detachment, presence of intraocular hemorrhage, presence of endophthalmitis, primary surgical repair combined with IOFB removal and the occurrence of postoperative complications. Combined phacoemulsification, vitrectomy, foreign-body extraction, and IOL implantation have become more and more popular in the management of such patients [1, 10-12]. However, small ferrous IVFD can be successfully removed by external magnetic extraction through the pars plana incision in patients without endophthalmitis and retinal injury [7]. Secondary removal of traumatic cataract combined with IOL implantation without PPV has been reported [8]. In this study, we explore the possibility of minimal surgery in selected patients by primary removal of small ferrous IVFD by external magnetic extraction and secondary removal of traumatic cataract by phacoemulsification and IOL implantation without PPV, and obtain good visual outcomes without postoperative complications.

Advances in vitreoretinal instruments and surgical techniques have improved the success of treatment in eye injuries with posterior segment IOFBs. Removal of posterior segment IOFBs by PPV is the main surgical procedure that provides direct viewing and controlled surgery [8]. However, removal of the posterior hyaloid, an important surgical goal, is difficult in relatively young patients. Various potential complications of PPV have been reported, including iatrogenic retinal tears, suprachoroidal hemorrhage, hypotony, choroidal detachments, wound leaks, vitreous incarceration and drop of foreign body on the macula $[4,9,13]$. In selected patients with small ferrous foreign body positioned in the vitreous cavity and without endophthalmitis and retinal injury, removal of the IVFD by external magnetic extraction through the pars plana incision may obtain good outcomes without complications [7]. In this study, all of IVFDs were successfully removed by external magnetic extraction without complications.

Lenticular injury as a result of an IOFB may occur directly if the foreign body passes through the lens. Removal of the IOFB by PPV in the presence of traumatic cataract and associated retinal pathology is difficult. To allow clear visualization of the posterior segment, cataract extraction under such circumstance is often necessary. However, in a few cases, a minor injury to the lens may result in a localized nonprogressive lens opacity that does not require surgery. Small IOFB with limited capsular damage may lead to self-limited lens injury [14], and spontaneous resolution of a traumatic cataract after removal of an intralenticular foreign body has been reported [15]. In one of our patients, traumatic lens opacity was mostly resolved and did not interfere with the visual axis after removal of a ferrous IVFD by external magnetic extraction, and the lens was preserved (unpublished data). 
In the absence of lens material into the anterior chamber in traumatic cataract, which may cause increased IOP or severe inflammatory reaction, some studies state that it is better to treat the eye with topical steroids to control inflammation first and to allow the capsule fibrosis. Delaying surgery would have allowed a more favorable implantation of the IOL in capsular bag with a better visual prognosis and lesser complications.

In this study, after primary removal of the IVFD, patients received systemic and topical antibiotic to prevent infection and topical steroids to control inflammation. Secondary cataract removal by phacoemulsification and IOL implantation in the capsule bag were successfully performed in 10 patients without significant enlargement of posterior capsule rupture. In 2 patients with relatively large foreign bodies, enlargement of the posterior rupture was inevitable, thus, anterior vitrectomy was performed and IOLs were placed in the ciliary sulcus. During follow-up, BCVA significantly improved, ranging from $20 / 32$ to $20 / 20$, and the IOLs were well centered. Complications, such as secondary glaucoma, endophthalmitis and retinal detachment were not observed.

\section{Conclusions}

In conclusion, in selected penetrating eyes with small ferrous IVFD combined with traumatic cataract without retinal injury, secondary glaucoma and endophthalmitis, primary removal of the IVFD by external magnetic extraction followed by secondary cataract removal and IOL implantation is an appropriate choice. Minimal surgery may obtain good visual outcomes without complications.

\section{Abbreviations}

BCVA: Best-corrected visual acuity; IOFB: Intraocular foreign body; IOL: Intraocular lens; IOP: Intraocular pressure; IVFD: Intravitreal foreign body; PPV: Pars plana vitrectomy

\section{Acknowledgements}

None.

\section{Funding}

This study was supported by Natural Science Foundation of Zhejiang Province LY15H120001 and National Natural Science Foundation of China (Grant No. 81370019 and 81870624). The funding bodies did not have a role in the study design, data collection, data analysis, interpretation of data, writing the manuscript, the critical revision and approval of submission of the current study.

Availability of data and materials

The datasets used and analyzed during the current study are available from corresponding author on reasonable request.

\section{Authors' contributions}

ZTS collected the data and drafted the manuscript. PPY and JJL collected the data. LZ participated in diagnosis and treatment of the patient. XDH guided the study and revised the manuscript. All authors have read and approved the final manuscript.

\section{Ethics approval and consent to participate}

This study has been performed in accordance with the Declaration of Helsinki and was approved by the Ethics Committee of the Second Affiliated Hospital, School of Medicine, Zhejiang University.

\section{Consent for publication}

Written informed consents were obtained from all subjects.

\section{Competing interests}

The authors declare that they have no competing interests.

\section{Publisher's Note}

Springer Nature remains neutral with regard to jurisdictional claims in published maps and institutional affiliations.

Received: 3 November 2018 Accepted: 7 February 2019

Published online: 19 February 2019

\section{References}

1. Dhoble P, Khodifad A. Combined cataract extraction with pars Plana vitrectomy and metallic intraocular foreign body removal through Sclerocorneal tunnel using a novel "magnet handshake" technique. Asia Pac J Ophthalmol (Phila). 2018;7(2):114-8.

2. Mahapatra SK, Rao NG. Visual outcome of pars plana vitrectomy with intraocular foreign body removal through sclerocorneal tunnel and sulcusfixated intraocular lens implantation as a single procedure, in cases of metallic intraocular foreign body with traumatic cataract. Indian J Ophthalmol. 2010;58(2):115-8.

3. Vatavuk Z, Pentz A. Combined clear cornea phacoemulsification, vitrectomy, foreign body extraction. and intraocular lens implantation CROAT MED J. 2004:45(3):295-8.

4. Albrieux M, Rouberol F, Bernheim D, Romanet JP, Chiquet C. Comparative study of 23-gauge vitrectomy versus 20-gauge vitrectomy for the treatment of rhegmatogenous retinal detachment. Graefes Arch Clin Exp Ophthalmol. 2011;249(10):1459-68.

5. Arumi JG, Boixadera A, Martinez-Castillo V, Corcostegui B. Transconjunctival sutureless 23-gauge vitrectomy for diabetic retinopathy. Review. Curr Diabetes Rev. 2009;5(1):63-6.

6. Eckardt C. Transconjunctival sutureless 23-gauge vitrectomy. Retina. 2005;25(2):208-11.

7. Kuhn F, Morris R. Posterior segment intraocular foreign bodies: management in the vitrectomy era. OPHTHALMOLOGY. 2000;107(5):821-2.

8. Pandey SK, Ram J, Werner L, Brar GS, Jain AK, Gupta A, Apple DJ. Visual results and postoperative complications of capsular bag and ciliary sulcus fixation of posterior chamber intraocular lenses in children with traumatic cataracts. J Cataract Refract Surg. 1999;25(12):1576-84.

9. Yuksel K, Celik U, Alagoz C, Dundar H, Celik B, Yazici AT. 23 gauge pars plana vitrectomy for the removal of retained intraocular foreign bodies. BMC Ophthalmol. 2015;15:75

10. Lam DS, Tham CC, Kwok AK, Gopal L. Combined phacoemulsification, pars plana vitrectomy, removal of intraocular foreign body (IOFB), and primary intraocular lens implantation for patients with IOFB and traumatic cataract. Eye (Lond). 1998;12(Pt 3a):395-8.

11. Batman C, Cekic O, Totan Y, Ozkan SS, Zilelioglu O. Combined phacoemulsification, vitrectomy, foreign-body extraction, and intraocular lens implantation. J Cataract Refract Surg. 2000;26(2):254-9.

12. Oztas Z, Nalcaci S, Afrashi F, Erakgun T, Mentes J, Degirmenci C, Akkin C. Posterior segment intraocular foreign bodies: the effect of weight and size, early versus late vitrectomy and outcomes. Ulus Travma Acil Cerrahi Derg. 2015;21(6):496-502.

13. Narayanan R, Tibra N, Mathai A, Chhablani J, Kuppermann BD. Sutureless 23-gauge versus 20-gauge vitrectomy with silicone oil injection in rhegmatogenous retinal detachment. Retina. 2012;32(5):1013-6.

14. Pieramici DJ, Capone AJ, Rubsamen PE, Roseman RL. Lens preservation after intraocular foreign body injuries. OPHTHALMOLOGY. 1996;103(10):1563-7.

15. Rofagha S, Day S, Winn BJ, Ou Jl, Bhisitkul RB, Chiu CS. Spontaneous resolution of a traumatic cataract caused by an intralenticular foreign body. J Cataract Refract Surg. 2008;34(6):1033-5. 